\title{
Effect of biological larviciding with Bacillus thuringiensis israelensis for malaria control on non- target vector mosquito species in rural Burkina Faso - A cluster randomized trial
}

Peter Dambach ( $\square$ peter.dambach@web.de )

Heidelberg University https://orcid.org/0000-0002-1086-6866

Till Bärnighausen

Ruprecht Karls Universitat Heidelberg Medizinische Fakultat Heidelberg

Anges Yadouleton

Centre de Recherche Entomologique de Cotonou

Martin Dambach

Universitat zu Koln

Issouf Traore

Centre de Recherche en Sante de Nouna

Patricia Korir

Rheinische Friedrich-Wilhelms-Universitat Bonn Medizinische Fakultat

Saidou Ouedraogo

Centre de Recherche en Sante de Nouna

Ali Sié

Centre de Recherche en Sante de Nouna

Rainer Sauerborn

Ruprecht Karls Universitat Heidelberg Medizinische Fakultat Heidelberg Norbert Becker

KABS

Valérie Louis

Ruprecht Karls Universitat Heidelberg Medizinische Fakultat Heidelberg

Research article

Keywords: biological larviciding, Bti, vector borne diseases, West-Africa

Posted Date: October 15th, 2019

DOI: https://doi.org/10.21203/rs.2.15770/v1 
License: (c) (i) This work is licensed under a Creative Commons Attribution 4.0 International License. Read Full License 


\section{Abstract}

Background: Biological larviciding is an additional tool that can help address the current dilemma in malaria control, namely vector resistances to pyrethroids and shifting of biting activity to times when people are not protected. Although malaria interventions primarily target Anopheles mosquitoes, there might be an impact on populations of other mosquito genera that share the same breeding sites. In this study we research to what extent Culex and Aedes mosquitoes, the primary vectors of numerous zoonotic diseases, are affected by larviciding interventions against malaria mosquitoes.

Methods: We researched the impact of different larviciding choices with Bacillus thuringiensis israelensis on non-target mosquitoes in 127 rural villages and a semi-urban town in a health district in Northwestern Burkina Faso. All villages were distributed into a total of three study arms with different larviciding choices: full, selective and untreated control. Geographically close villages were distributed into clusters to avoid contamination between treated and untreated villages. Adult mosquitoes were captured in light traps inside and outside houses during the rainy seasons of a baseline and an intervention year. $\mathrm{A}$ negative binomial regression was used to determine the reductions achieved through larviciding among different mosquito genera.

Results: Larviciding interventions against malaria showed only limited or no impact against Culex mosquitoes, while against Aedes, reductions of up to $34 \%$ were achieved when all detected breeding sites in the public space were treated. While the semi-urban setting showed high abundance of Culex, in the rural villages we captured more Aedes.

Conclusions: Future larviciding programs should be evaluated for including the treatment of Aedes and Culex breeding habitats. Since the major cost components of such programs are labor and transport, other disease vectors could be targeted at little additional cost.

\section{Introduction}

Larval source management, the elimination, alteration and treatment of breeding grounds of disease transmitting mosquitoes, has been performed for centuries. During the 1950s, insecticides had become a promising tool to pursue the goal of global malaria eradication. Today's malaria vector control is predominantly targeting the adult stages of mosquitoes through bed nets and indoor residual spraying. While the larviciding approach evolved through the introduction of biological substances that are not harmful for the environment, its routine implementation is predominantly performed in high income countries. Several large scale trials that have shown technical feasibility impact on vector populationswere carried out in the resource restraint setting of urban and rural Africa (1-4). However, for African settings, no reduction in malaria incidence was observed to date (5), and in some settings, such as extensive floodplains, biological larviciding alone seems not to be a recommendable stand-alone approach (6). Promising results were achieved with the bacterial toxins Bacillus thuringiensis israelensis 
(Bti) and Bacillus sphaericus (Bs),, that act selectively against mosquitoes and are environmentally sound (7).

Although the primary-target during anti-malarial larviciding interventions are mosquitoes from the genus Anopheles, there is an impact on other mosquito genera that inhabit the same breeding sites as Anopheles. Within the study region in North-Western Burkina Faso the typical malaria mosquito breeding sites consist of water holes, brick-works, small ponds, wet rice fields and large, flooded areas $(8,9)$. During the peak rainy season puddles can persist up to several weeks and equally allow for mosquito breeding. Apart from a wide variety of Anopheles species, those breeding sites are equally attractive for oviposition to female Culex mosquitoes and there is evidence that, despite observed inter species predation (10), both, Anopheles spp. and Culex spp.are more likely to coexist in the same breeding sites than would be expected by chance alone $(11,12)$. While there is a major overlap in breeding site preference between Anopheles and Culex mosquitoes, Culex were found to be generally more successful breeding in heavily polluted water bodies $(13,14)$. Within the study region, those heavily polluted sites predominantly prevailed in the semi-urban town of Nouna, mainly as septic tanks and dirty puddles, while they were almost absent in the rural villages. Aedes mosquitoes on the other hand normally prefer other types of breeding sites that are not a primary target during larviciding interventions against malaria. Typical breeding sites of the regionally common vectors Aedes albupictus and $A$. aegypti include drinking water containers, clay jars, tin cups, car tires and other small objects that can harbor rainwater $(15,16)$.

Although the highly abundant Culex and Aedes mosquitoes are not capable of transmitting malaria, they do bear increasing public health relevance in Africa through the transmission of several arboviral and parasitic infections. Culex mosquitoes are known to transmit West-Nile fever (Culex pipiens),, Sindbisvirus (C. pipiens, C. univittatus) and parasitic nematodes such as Wucheria bancrofti and Brugia malayi, the cause of lymphatic filariasis. Several species from the genus Aedes are known to transmit the dengue, Chikungunya and yellow fever viruses. Both, Culex and Aedes are capable of transmitting the Zika virus. To date there are numerous mosquito-borne arboviruses transmitted by Culex and Aedes mosquitoes that are indigenous to Africa, and several of them are likely to receive greater geographical distribution and medical importance with increasing population growth, travel and deforestation (17). Mansonia do bear public health relevance as some species are capable to transmit lymphatic filariasis (18).

In the light of high vector competence and capacity for transmitting several emerging diseases of at least some of these genera, it is important to know how much they are affected by malaria vector control interventions. In this study we research to what extent non-malaria mosquito populations are impacted by Bti based larviciding against malaria vectors in the public space in and around 127 rural villages and a semi-urban town in North-Western Burkina Faso. Furthermore, we discuss possible health co-benefits of larviciding based malaria vector control on other common tropical vector borne diseases.

\section{Methods}


- Study area

The study area consisted of all 127 rural villages and the semi-urban town that were part of the extended health district of the Kossi region in Northwestern Burkina Faso, close to the Mali border. It stretched over a total surface of about $4,770 \mathrm{~km}^{2}$ and covered some 156,000 inhabitants. The study area is heterogeneous regarding its ecology. While the Northern parts towards the Sahel often feature sandy soils with high infiltration rates and lower numbers of environmental mosquito breeding sites, the picture is different in the South where there are more stagnant water bodies and wet rice growing areas. The eastern border of the district is characterized by wetlands around the Sourou valley.

- Study design

The study was designed as a cluster randomized trial, administering different larviciding choices to mosquito breeding sites. Reporting followed the CONSORT guidelines for randomized trials where applicable. Three larviciding choices (i: untreated control, ii: treatment of all breeding sites and iii: risk map based larvicide application to only the most productive breeding sites) were performed within a total of 9 village clusters (Figure 1). Villages were clustered to avoid spill-over effects caused by the flight range of mosquitoes $(19,20)$. Three clusters always represented areas that were similar in surface water availability, soil type, vegetation and other geographical factors (ecozone). Larviciding choices were randomly assigned to the predefined clusters, with the criterion to have each larviciding choice represented in each geographical ecozone (figure 1). The study comprised three years, a baseline year without intervention (2013) and two intervention years $(2014+2015)$. Here we present results from the baseline and the first intervention year, in which the abundance of non-anophelines was seized. Larviciding with Bti VectoBac® WG, AM65-52 strain (Valent BioSciences Corporation, IL, USA) was performed during and after the rainy season in the public space in and around villages but not in private compounds. VectoBac ${ }^{\circledR}$ was diluted in pond water, that was filtered through cotton cloth and brought out onto the water surface using inox steel knapsack sprayers (Mesto®, Freiberg, Germany). Prior to the intervention we identified the optimum dosages for field application (21). Maps with all publicly accessible water bodies were generated during field visits for villages with exhaustive larviciding. For villages that received guided treatment, remote sensing derived risk maps for larval productivity were used (9).

- Adult mosquito monitoring

The primary outcome to assess larviciding efficacy was the abundance of different mosquito species. For the collection of adult mosquitoes Center for Disease Control light traps (Model 512, John W. Hock Company, Gainesville, Florida) were used. Indoor and outdoor captures were performed in 27 villages in 2013, and in 36 villages in 2014; additionally, the seven town quarters of Nouna were included. Light trap captures were performed every two weeks, following a rotating system with two independent fieldwork teams, covering 4 villages per night, resulting in a total of at least 10 sample rounds per village per rainy season. 
Per village, three places were chosen regarding their central position in the village and in agreement with the household head, where a light trap each was positioned about one meter above the ground. Light traps were installed in a distance of approximately 100 to 150 meters from each other to detect possible local differences in vector abundance between different places within one village. The traps inside houses were installed near the sleeping places equipped with untreated bed nets, the traps outside were put beside the house within the common courtyard, where people sat in the evenings. Mosquitoes were collected between 18:00 and 06:00 hours to fully cover the peak biting period. Species determination was performed using microscopes, following the WRBU (Walter Reed Biosystematics Unit) identification keys (22).

- Statistical analysis

Statistical analysis was performed using Stata/IC 14.2 for Windows (StataCorp LLC, 4905 Lakeway Drive, College Station, TX 77845, USA). Count of female mosquitoes collected per night per trap was used as the outcome variable. A negative binomial regression (Stata function "nbreg"), corresponding to a generalization of a Poisson distribution, was performed to model the count data, which showed overdispersion. The random effect was integrated at village level.

\section{Results}

- Mosquito genera distribution before and during larviciding

A total of 36148 female mosquitoes were caught within light traps between September and December 2013 and between June and November 2014. During the four months of sampling in the pre intervention year, 12,073 female mosquitoes were caught, of which 5,842 (48\%) were Culex spp. (Linnaeus), 3,677 (30\%) Anopheles spp. (Meigen) and 2,317 (19\%) Aedes spp. (Meigen) and 237 (2\%) Mansonia spp. (Blanchard). During the six month of mosquito collections in the intervention year, 24,075 female mosquitoes were captured; the share of Culex mosquitoes on the total catch increased to $55 \%(13,205)$, while the abundance of Anopheles decreased to 23\% $(5,345)$. The share of Aedes remained almost unchanged with $22 \%(5,357)$ of the total catch while that of Mansonia decreased to $0.7 \%(168)$. Given the small number of Mansonia mosquitoes captured, the statistical analysis focuses on the most abundant genera. Figure 1 illustrates the geographical variation in Culex and Aedes mosquito numbers among villages and in the semi-urban town of Nouna during the annual period of high mosquito abundance. The natural variations of other mosquito genera (Culex, Aedes, Mansonia) were not linked to those of Anopheles, which showed an increase in the second study year. In the untreated control group, the presence of other genera declined compared to the baseline year.

- Outdoor and indoor captures

Anopheles mosquitoes were captured significantly more often indoors than outdoors $(p<0.001)$ with a 61 $\%$ average; this was the largest proportion among the genera. Culex and Aedes mosquitoes were also predominantly captured indoors ( $54 \%, p=0.026$ and $57 \%, p=0.071$, respectively) but the difference 
between outdoor and indoor was less pronounced; it was significant only for Culex mosquitoes and there was a large variability for Aedes mosquitoes The much fewer Mansonia mosquitoes, however, were largely captured outdoors $(75 \%, p=0.007)$. In addition, Figure 2 shows the mosquito abundance by genus in the different treatment areas. In the semi-urban area of Nouna, Culex mosquitoes were highly abundant despite being in an area of full treatment.

\section{Figure 1 about here}

\section{Figure 2 about here}

- Reduction of non-anophelines through Bti-spraying

Figure 3 shows that environmental larviciding with Btionly had no significant effect on the abundance of Culex mosquitoes $(1.06,95 \% \mathrm{Cl}: 0.96-1.16$ and $0.96,95 \% \mathrm{Cl}: 0.88-1.06$, for guided and full treatment respectively) while the abundance of Aedes mosquitoes was significantly reduced by $34 \%$ with the full treatment $(0.66,95 \% \mathrm{Cl}: 0.57-0,76)$ but not with the guided treatment $(0.94,95 \% \mathrm{Cl}$ : $0.85-1.05)$.

\section{Figure 3 about here}

- Mosquito abundance over time

The abundance of Culex and Aedes mosquitoes followed the course of the rainy season. Culex populations began to rapidly grow with a lagged onset of about two weeks after the first rains in July, reaching a maximum in August and then slowly declined until the end of the capture period in end November (Figure 4). Fully, guided and untreated rural areas showed similar abundance patterns of Culex during the rainy season, underlining the absence of a significant impact of larviciding on this genus at any point in time observed. Despite larviciding interventions having been in place with the first rains in beginning July, Culex catches peaked at almost 16 mosquitoes per night per trap in August. For Aedes the picture is inverse, the larviciding interventions showed greatest impact during the month of August and the highest reductions achieved were registered in the semi-urban setting of Nouna town, where catches were as low as 0.5 mosquitoes per night per trap. However, towards the later months September and October, the number of Aedes did rise again.

\section{Figure 4a (Culex) and 4b (Aedes) about here}

\section{Discussion}

While the abundance of adult Anopheles spp.was suppressed by up to $70 \%$ (23), the same larviciding intervention did show only very limited impact on the abundance of Culex spp. mosquitoes. Reductions of Aedes spp. were low in the guided treatment arm but reached $34 \%$ in the full treatment arm. The 
recorded mosquito reductions differed depending on whether the captures were performed at indoor or outdoor posts. While for Anopheles, the reductions at indoor capture post were twice as high compared to those from outdoor posts (23), the effect on non-Anopheles showed the inverse picture, with twice the reductions achieved at outdoor posts. It is difficult to conclude what led to the higher Anopheles reductions at outdoor posts. One possible contributor to this result might be unknown characteristics of the study villages. A previous study in the region that used human landing catches found Anopheles gambiae s.l. to be the predominant species with a share of more than 90 percent of the total Anopheles catch (24). However, species or sub-species does not seem to be a determinant for being attracted to either outdoor or indoor LTC posts and was found to vary between different geographical locations (25). Despite possible differences in species composition and LTC trap preference between different villages, those are unlikely to explain differences in achieved reductions between outdoor and indoor LTC posts. Reductions achieved through targeting mosquito larvae would be expected to appear uniformely, unless different mosquito species are attracted differently to outdoor and indoor LTC posts and larviciding intervention affected different species differently. The use of light traps seemed to be more effective in capturing indoor resting Culex, Aedes and Anopheles mosquitoes, while the traps positioned outdoors showed generally lower mosquito numbers. This contrasts with another study in the same area that used human landing catches and found the abundance of all three genera between indoor and outdoor to be roughly the same (24).

The minimal impact of larviciding activities on Culex mosquitoes compares to findings from Dar es Salaam in Tanzania, where only little effect of larviciding on adult Culexwas achieved, while the primary target Anopheles was strongly impacted (1). Culex are known to breed even in heavily polluted water (26) and we observed the same within the survey region, where heavily polluted oviposition sites such as septic tanks, oily puddles and open surface toilet were exclusively populated with Culex larvae. Since those breeding sites were mostly situated within private courtyards, they were not included in spraying activities in the public space. On the other hand, Culex mosquitoes did not show the breeding site exclusivity of Anopheles spp. but were present in most of the breeding sites in the region (Dambach unpublished data, collected during fieldwork for (9)).

Theoretically, difference in larviciding success between different mosquito genera might not only be ascribed to different habitat types but as well to their individual susceptibility to Bti. However, reports on the effectiveness of $B t i$ on the larvae of different genera within the Culicidae family differ. While some studies reported higher susceptibility of Culex towards Bti (27-30), others found that Anopheles and Aedes required lower lethal Bti concentrations $(31,32)$. Pollution and eutrophication of breeding sites is known to influence the effectiveness of Bti(33). If breeding in sites with increased pollution, they might have been less affected by spray activities.

\section{Strengths and limitations}


The conclusiveness of this study benefits from the large spatial and temporal extent of larviciding activities in 127 rural villages and a semi-urban town and the high amount and collection frequency of entomological data, which is extensive compared to many other studies. To our knowledge this study is the first to systematically evaluate the impact of larviciding against malaria vectors on other disease transmitting mosquito genera. There are also limitations to this study. Mosquito collections in 2013 started later than initially planned and data is available from September on only, resulting in a relatively short overlap period of three months with the mosquito sampling of the following intervention year. Treatment arms were randomized at the level of village clusters. Although this does not follow the standard approach for a randomized control trial of medical studies, it was the best possible approach in a geographical and environmental context. Mosquitoes do not only bite in the immediate vicinity of their breeding grounds but are able to cover distances of several kilometers during their search for blood meal, hence, we applied larviciding over a larger area to avoid infiltration of mosquitoes from untreated areas. For this reason, villages in which the same larviciding approach was applied were clustered geographically.

\section{Importance for vector control programs}

The findings presented here have several implications for the implementation of larviciding programs. Although in the context of malaria, other mosquito species have less importance, they do play a role when it comes to nuisance and vector borne diseases such as lymphatic filariasis, yellow fever, dengue, and Zika. Although malaria control programs that only perform larviciding in the public space, as the one presented here, can provide major reductions in Anopheles mosquito abundance, they lack the ability to sufficiently reduce other disease transmitting mosquitoes such as Culex and Aedes that develop in breeding sites that are typically found in private compounds (34). While we expect only limited additional relief in Anopheles abundance when extending spraying activities to private compounds, it might show a strong impact on the numbers of Culex and Aedes mosquitoes. In the study area breeding sites of both genera were predominantly found within courtyards, particularly in the semi-urban setting of Nouna town.

\section{Conclusion}

In the wake of the introduction of other vector borne diseases to Africa, such as dengue, it could become important to not limit vector control efforts to Anopheles, but extend them to Culex and Aedes and others as well. Since the major cost components of larviciding based vector control programs are infrastructural and personnel expenditures, it could be beneficial to bundle efforts for controlling malaria, dengue and other mosquito-borne diseases into an integrated program where necessary.

\section{Abbreviations}

Bti - Bacillus thuringiensis israelensis

Bs - Bacillus sphaericus 


\section{Declarations}

- Competing interests

The authors declare that they have no competing interests

- Authors' contributions

P. D. and R.S and N. B. developed the concept and design of the study. I. T., P. D. and S. O. supervised and performed the collection of the data. P. D. V. L. performed the analysis and interpretation of the data. V.L and T. B. ran the statistical analyses in STATA. A. Y., M. D., P. K., and N. B. contributed to the entomological and ecological interpretation of the data and to the discussion. P. D. and V. L. wrote the paper. All authors contributed to writing of the paper and the interpretation of the data. All authors read and approved the final manuscript.

- Acknowledgments

We are deeply thankful to the communities for their support and willingness to participate in this research. We are also grateful to the field and laboratory staff at the research facility in Nouna for their valuable work and commitment to make the project successful and evolving.

- Funding

This study was funded by the Manfred Lautenschläger foundation, Wiesloch, Germany. The funder did not have any role in the design implementation and the analysis of the study.

- Ethics approval and consent to participate

This study was approved by the ethics committees of the University of Heidelberg in Germany, the national ethics board of Burkina Faso in Ouagadougou and the local ethics committee at the research site in Nouna. Aggregated collective informed consent for the spraying activities was collected for each village through the traditional village chiefs. The population was invited at a central place in the village and the project, its goals and involved activities were explained in local language. In the following, public discussions were held with the opportunity to ask questions or express concern. During the intervention there was additional community sensitization and information performed through the local radio station. The study was registered under the trial id PACTR201611001721299 on the Pan African Clinical Trials Registry (https://pactr.samrc.ac.za).

- Consent for publication

There are no case presentations that require disclosure of respondents' confidential data/information in this study. 
- Availability of data and material

The datasets supporting the conclusions of this article are available at the Health Research Center in Nouna, Burkina Faso and will be made easily available on request, when required.

\section{References}

1. Geissbühler Y, Kannady K, Chaki PP, Emidi B, Govella NJ, Mayagaya V, u. a. Microbial Larvicide Application by a Large-Scale, Community-Based Program Reduces Malaria Infection Prevalence in Urban Dar Es Salaam, Tanzania. PLoS ONE. 31. März 2009;4(3):e5107.

2. Fillinger U, Lindsay SW. Suppression of exposure to malaria vectors by an order of magnitude using microbial larvicides in rural Kenya. Trop Med Int Health. November 2006;11(11):1629-42.

3. Dambach P, Traoré I, Becker N, Kaiser A, Sié A, Sauerborn R. EMIRA: Ecologic Malaria Reduction for Africa-innovative tools for integrated malaria control. Glob Health Action. 5. November 2014;7(10).

4. Maheu-Giroux M, Castro MC. Impact of Community-Based Larviciding on the Prevalence of Malaria Infection in Dar es Salaam, Tanzania. PLOS ONE. 14. August 2013;8(8):e71638.

5. Tusting LS, Thwing J, Sinclair D, Fillinger U, Gimnig J, Bonner KE, U. a. Mosquito larval source management for controlling malaria. Cochrane Database Syst Rev. 2013;8:CD008923.

6. Majambere S, Lindsay SW, Green C, Kandeh B, Fillinger U. Microbial larvicides for malaria control in The Gambia. MalarJ. 2007;6(1475-2875 (Electronic)):76.

7. Siegel JP. The Mammalian Safety of Bacillus thuringiensis- Based Insecticides. J Invertebr Pathol. 1. Januar 2001;77(1):13-21.

8. Dambach P, Sié A, Lacaux J-P, Vignolles C, Machault V, Sauerborn R. Using high spatial resolution remote sensing for risk mapping of malaria occurrence in the Nouna district, Burkina Faso. Glob Health Action. 2009;2(10).

9. Dambach P, Machault V, Lacaux J-P, Vignolles C, Sié A, Sauerborn R. Utilization of combined remote sensing techniques to detect environmental variables influencing malaria vector densities in rural West Africa. Int J Health Geogr. 2012;11(8).

10. Muturi EJ, Kim C-H, Jacob B, Murphy S, Novak RJ. Interspecies Predation Between Anopheles gambiae s.s. and Culex quinquefasciatus Larvae. J Med Entomol. 1. März 2010;47(2):287-90.

11. Muturi EJ, Mwangangi J, Shililu J, Jacob BG, Mbogo C, Githure J, u. a. Environmental factors associated with the distribution of Anopheles arabiensis and Culex quinquefasciatus in a rice agroecosystem in Mwea, Kenya. J Vector Ecol. 1. Juni 2008;33(1):56-63.

12. Kweka EJ, Zhou G, Beilhe LB, Dixit A, Afrane Y, Gilbreath TM, u. a. Effects of co-habitation between Anopheles gambiae s.s. and Culex quinquefasciatus aquatic stages on life history traits. Parasit Vectors. 9. Februar 2012;5:33.

13. Munga S, Minakawa N, Zhou G, Mushinzimana E, Barrack O-OJ, Githeko AK, u. a. Association between land cover and habitat productivity of malaria vectors in western Kenyan highlands. Am J 
Trop Med Hyg. Januar 2006;74(1):69-75.

14. Mwangangi JM, Muturi EJ, Shililu J, Muriu SM, Jacob B, Kabiru EW, u. a. Contribution of different aquatic habitats to adult Anopheles arabiensis and Culex quinquefasciatus (Diptera: Culiddae) production in a rice agroecosystem in Mwea, Kenya. J Vector Ecol. 2008;33(1):129-38.

15. Madeira NG, Macharelli CA, Carvalho LR. Variation of the Oviposition Preferences of Aedes aegypti in Function of Substratum and Humidity. Mem Inst Oswaldo Cruz. April 2002;97(3):415-20.

16. Simard F, Nchoutpouen E, Toto JC, Fontenille D. Geographic Distribution and Breeding Site Preference of Aedes albopictus and Aedes aegypti (Diptera: Culicidae) in Cameroon, Central Africa. J Med Entomol. 1. September 2005;42(5):726-31.

17. Braack L, Almeida APG de, Cornel AJ, Swanepoel R, Jager C de. Mosquito-borne arboviruses of African origin: review of key viruses and vectors. Parasit Vectors. 2018;11.

18. Ughasi J, Bekard HE, Coulibaly M, Adabie-Gomez D, Gyapong J, Appawu M, u. a. Mansonia africana and Mansonia uniformis are Vectors in the transmission of Wuchereria bancrofti lymphatic filariasis in Ghana. Parasit Vectors. 7. Mai 2012;5(1):89.

19. Ejercito A, Urbino M. Flight range of gravid and newly emerged Anopheles. Bull World Health Organ. 1951;(3):663-71.

20. Quraishi MS, Esghi N, Faghih MA. Flight range, lengths of gonotrophic cycles, and longevity of P-32labeled Anopheles stephensi mysorensis. J EconEntomol. Februar 1966;59(0022-0493 (Print)):505.

21. Dambach P, Louis VR, Kaiser A, Ouedraogo S, Sié A, Sauerborn R, u. a. Efficacy of Bacillus thuringiensis var. israelensis against malaria mosquitoes in northwestern Burkina Faso. Parasit Vectors. 15. August 2014;7(1):371.

22. Walter Reed Biosystematics Unit. Walter Reed Biosystematics Unit [Internet]. 2018 [zitiert 9. Januar 2017]. Verfügbar unter: http://www.wrbu.org/index.html

23. Dambach P, Bärnighausen T, Traore I, Ouedraogo S, Sie A, Sauerborn R, u. a. Reduction of malaria vector mosquitoes in a large scale intervention trial in rural Burkina Faso using Bti based larval source management. International Journal of Epidemiology, under review. 2019;

24. Dambach P, Schleicher M, Korir P, Ouedraogo S, Dambach J, Sié A, u. a. Nightly Biting Cycles of Anopheles Species in Rural Northwestern Burkina Faso. J Med Entomol. 2018;55(4):1027-34.

25. Degefa T, Yewhalaw D, Zhou G, Lee M, Atieli H, Githeko AK, u. a. Indoor and outdoor malaria vector surveillance in western Kenya: implications for better understanding of residual transmission. Malar J. 6. November 2017;16(1):443.

26. Kengne IM, Brissaud F, Akoa A, Eteme RA, Nya J, Ndikefor A, u. a. Mosquito development in a macrophyte-based wastewater treatment plant in Cameroon (Central Africa). Ecol Eng. 1. November 2003;21(1):53-61.

27. Marina CF, Bond JG, Muñoz J, Valle J, Novelo-Gutiérrez R, Williams T. Efficacy and non-target impact of spinosad, Bti and temephos larvicides for control of Anopheles spp. in an endemic malaria region of southern Mexico. Parasit Vectors. 2014;7(1):55. 
28. Dylo P, Martin C, Mhango C. Efficacy of Bacillus thuringiensis var israelinsis (Bti) on Culex and Anopheline mosquito larvae in Zomba. Malawi J Sci Technol. 1. Januar 2014;10(1):40-52-52.

29. Dominic Amalraj D, Sahu SS, Jambulingam P, Boopathi Doss PS, Kalyanasundaram M, Das PK. Efficacy of aqueous suspension and granular formulations of Bacillus thuringiensis (Vectobac) against mosquito vectors. Acta Trop. 25. März 2000;75(2):243-6.

30. Sharma SN, Shukla RP, Mittal PK, Adak T, Kumar A. Efficacy of a new formulation of Bacillus thuringiensis var israelensis (Bti) in laboratory and field conditions of Kumaun foothills of Uttaranchal, India. J Commun Dis. Dezember 2003;35(4):290-9.

31. Ch arles J-F, Nielsen-LeRoux C. Mosquitocidal bacterial toxins: diversity, mode of action and resistance phenomena. Mem Inst Oswaldo Cruz. 2000;95:201-6.

32. Kahindi SC, Midega JT, Mwangangi JM, Kibe LW, Nzovu J, Luethy P, u. a. Efficacy of Vectobac DT and Culinexcombi Against Mosquito Larvae in Unused Swimming Pools in Malindi, Kenya. J Am Mosq Control Assoc. Dezember 2008;24(4):538-42.

33. Margalit J, Bobroglo $\mathrm{H}$. The effect of organic materials and solids in water on the persistence of Bacillus thuringiensis var. israelensis Serotype H-141. J Appl Entomol. 1984;97(1-5):516-20.

34. Dambach P. New approaches for integrated and cost-effective malaria vector control. Journ Rare Dis Treat. 2018;3(1):6-10.

\section{Figures}




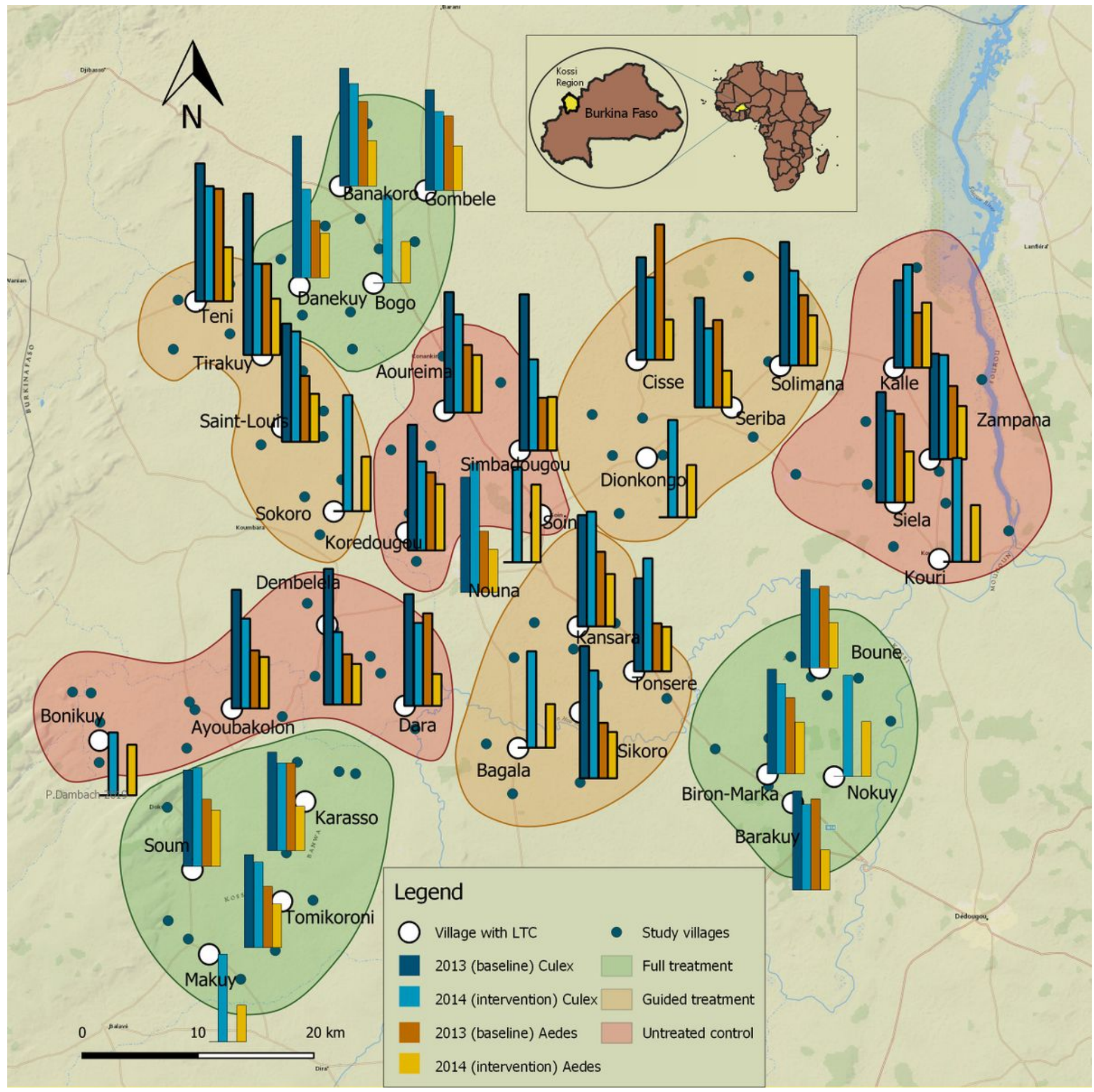

\section{Figure 1}

Mosquito genera distribution in baseline and intervention year. Study villages are shown with blue dots; villages with light trap captures (LTC) are marked with white dots. Bars show the average numbers of female Culex (blue color range) and female Aedes (orange color range) captured per trap per night indoors and outdoors in September and October 2013 and 2014. Colors of the cluster areas indicate treatment choice (green $=$ full treatment, orange = guided treatment, red = untreated control). In 2014, 9 additional villages were added to the mosquito collections. 


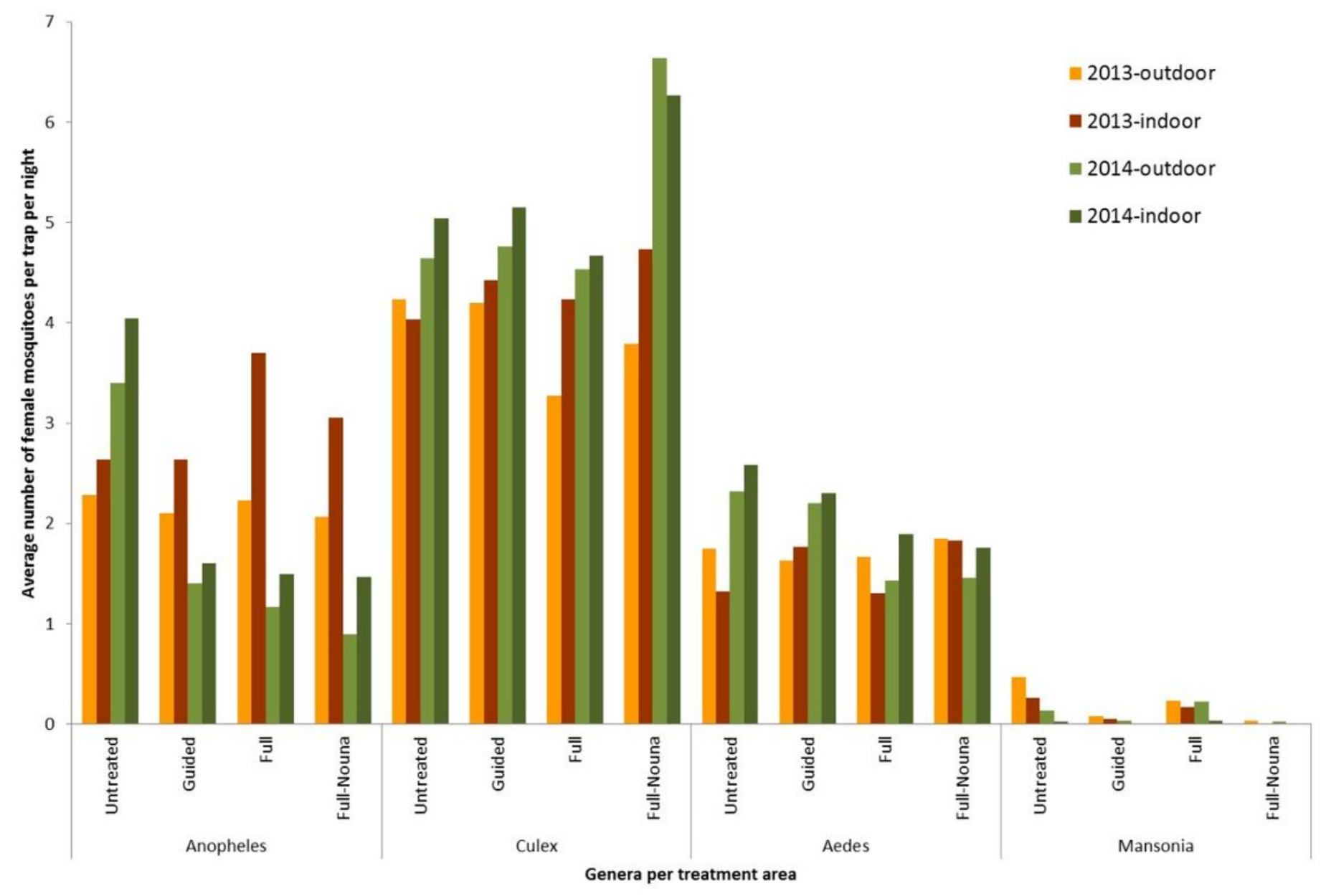

Figure 2

Average number of female mosquitoes per trap per night, per genus and treatment area for indoor and outdoor LTC capture over the collection period in 2013 and 2014 


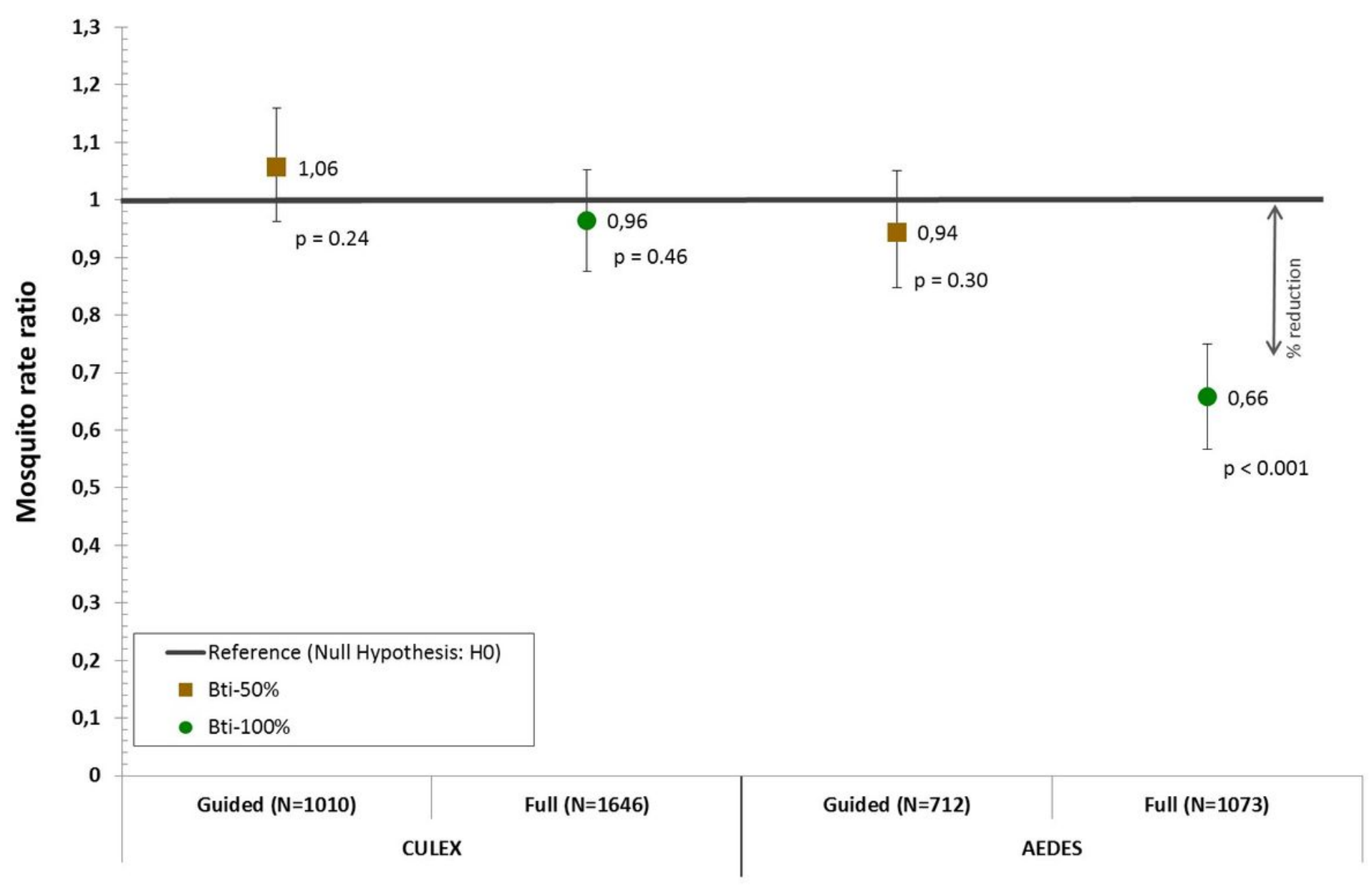

\section{Figure 3}

Point estimates of the regression model for the intervention year compared to the baseline year indicating the reduction in the counts of female Culex and Aedes mosquitoes achieved through different larviciding choices with Bti, i.e. guided (Bti-50\%) or full treatment (Bti-100\%) of Anopheles larval habitats treated in rural villages, excluding the town of Nouna. The reference line represents the rate ratio value under the null hypothesis: i.e. the count of female mosquitoes in the control areas receiving no Bti treatment are not significantly different from the counts in areas receiving a guided or full treatment Bti treatment. 


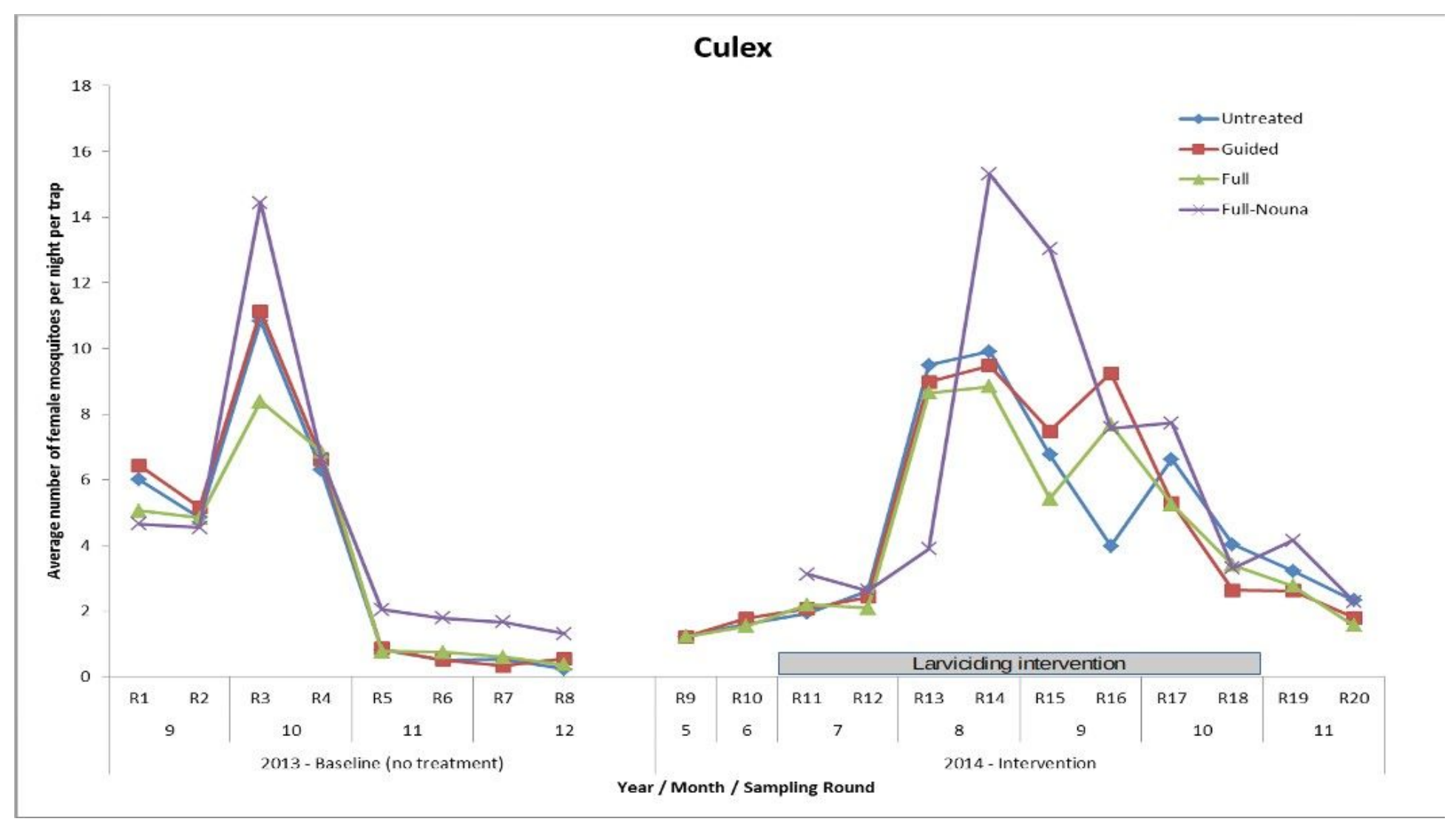

\section{Figure 4}

ime line of mosquito abundance (average number of female mosquitoes per trap per night) in the different Bti treatment areas, A) Culex mosquitoes B) Aedes mosquitoes

\section{Supplementary Files}

This is a list of supplementary files associated with this preprint. Click to download.

- CONSORT2010ChecklistDambach2019.pdf 